\title{
Signal strength monitoring in avalanche structures operated in Geiger mode
}

\author{
Josef Blažej ${ }^{1}$ \\ Czech Technical University in Prague \\ Brehova 7, 11519 Praha 1, Czech Republic \\ E-mail: blazejefjfi.cvut.cz \\ Ivan Procházka \\ Czech Technical University in Prague \\ Brehova 7, 11519 Praha 1, Czech Republic \\ E-mail: prochazkefjfi.cvut.cz
}

\begin{abstract}
We are presenting recent results of experimental analysis of avalanche build-up process in selected silicon avalanche structures. Structures has been operated in Geiger mode controlled by active gating and passive quenching circuit. Measurable changes in avalanche rapidity allow us to monitor optical signal strength in few-photon region $1-1000$ photons. The resolution, reproducibility and dynamical range of these shot-by-shot based measurements will be discussed. The presented types of measurements allows us to determine the photon number involved in the detection process simultaneously with standard photon counting using single active area. In compare with multi-pixel detector the effects of multiple quenching and coherent effects are not involved in experiments. In other hands, the current photon number resolution is in factor of 3 only.
\end{abstract}

International Workshop on New Photon Detectors (PD09)

Shinshu University Matsumoto Japan

24-26 June 2009

${ }^{1}$ Speaker 


\section{Introduction}

The avalanche photodiodes acting as photon counting devices has been pioneered by S. Cova and co-workers [1]. This detector, called Single Photon Avalanche Diode (SPAD), is an avalanche photodiode structure prepared using a conventional planar technology on silicon reported by Haitz [2]. Single photon sensitivity is achieved by biasing the diode above the junction breakdown voltage. In this stage the first absorbed photon is capable of triggering the avalanche multiplication of carriers; a fast rise-time current pulse is generated. The leading edge of the current pulse marks the event of the photon absorption with picosecond accuracy. The current increase is terminated by an external circuit connected to the diode, where the typical value of the gain achieved exceeds $1 \times 10^{9}$. Such an operation mode is called a Geiger mode. The operation of the detector may be controlled: gated, by an external electrical signal. The detector is activated short time before the arrival of the photon of interest.

In our experiments we have concentrated on the operation of the SPAD diodes in the regime of detecting single or several photons per pulse. The existing solid state photon counting detectors have been dedicated for high timing resolution and stability of single photon events. However, the high timing stability is maintained for individual single photons detection, only. If more than one photon is absorbed within the detector time resolution, the detection delay will be significantly affected. This fact is restricting the application of the solid state photon counters to cases where single photons may be guaranteed. The example of such an application is the time resolved spectroscopy, single molecules detection and similar. However, there is a demand for detection of optical pulses consisting of several up to several thousands of photons with picosecond resolution and stability. No conventional optical detector or photon counter can fulfill these requirements. The standard SPAD based photon counters work in a purely digital mode - uniform output signal is generated once a photon is detected. If the input signal consists of several photons, the first absorbed one triggers the avalanche. For multiple photon signals, the detection delay will be shorter in comparison to the single photon events. The detection delay dependence on the photon number is called the "detection time walk". To enable the detector operation in both the single and multiphoton signal regime with a minimal time walk, a time walk compensation technique has been developed [3] in nineties. The operation of this device was based on unique properties of the K14 SPAD detectors [4]. The procedure is based on the fact that the avalanche rise-time of the K14 SPAD chip is depending on the input optical signal strength. The circuit is using this information to compensate the time walk of the detector in this dynamical range.

The critical limitation of the technique described above is very small measured difference in an avalanche build up rise-time in the range of tens of ps only. That is why new effort has been directed to the avalanche build up studies. The availability of $\mathrm{GHz}$ bandwidth digital oscilloscopes digitizing at a speed of 40 Gsamples per second is opening new possibilities in an avalanche buildup studies. 


\section{Experiment}

The SPAD chips were tested and operated in an active gated and passively quenched circuit. The scheme enables to bias the chip to the voltage just below its breakdown voltage. The SPAD bias is increased for a short time (100 ns typically) for additional step of the negative gate pulse. The picosecond laser diode Hamamatsu C4725 providing $48 \mathrm{ps}$ wide pulses at $778 \mathrm{~nm}$ was used as a signal source. The laser output was attenuated by means of a stack of calibrated neutral density filters and focused on the detector active area. The precision X-Y-Z stage enabled us to investigate focusing and position sensitive effects of the detection process. The optical signal intensity was measured using the detection chip in a linear mode with unity gain and monitoring its reverse current by the electrometer Keithley $610 \mathrm{C}$ and operating the power stabilized laser source at a high repetition rate of $1 \mathrm{Mhz}$. The standard repetition rate of experiment was $1 \mathrm{kHz}$.

\section{Results}

The avalanche photodiode chip under test was the K14 structure made by our University. This is the "thin" SPAD structure on silicon, the active area diameter is 200 microns. The K14 type SPADs are characterized by several special features in comparison to other SPADs. It is the exceptionally high active area uniformity in both the timing jitter and mainly of the detection delay over an entire sensitive area [5]. The diode structure was kept at a room temperature and in darkness. The breakdown voltage is 30.0 Volts, diode was biased $5 \mathrm{~V}$ above. For strong optical signal ( $\sim 1000$ photons/pulse) substantially faster avalanche buildup and hence faster current risetime was observed. The avalanche buildup corresponding to single photon was observed to be close to exponential one as expected from the basic theory.

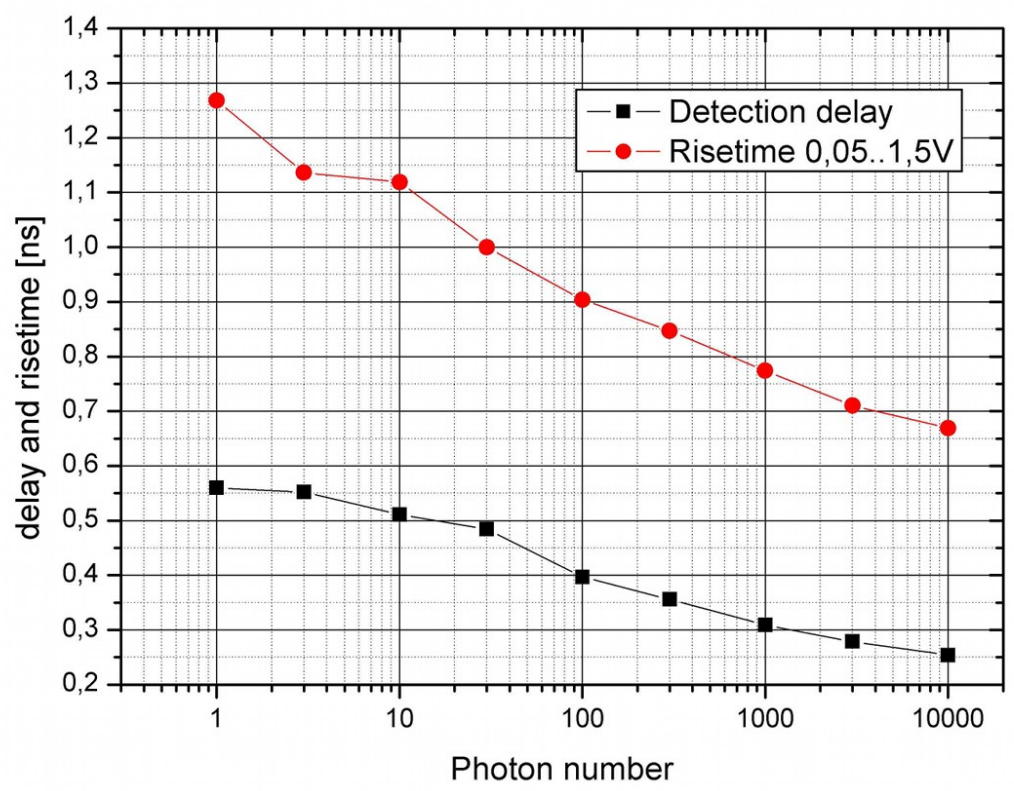

Figure 1: Correlation of the avalanche current risetime to the detection delay, K14 SPAD biased $5 \mathrm{~V}$ above its breakdown voltage, optical pulses $48 \mathrm{ps}$ long, trigger levels $10 \mathrm{mV}$ and $1.5 \mathrm{~V}$ optimized for minimum trigger jitter and maximum risetime difference. 
For higher photon counts, the avalanche buildup consists of two parts. In the initial part the current risetime is much faster than exponential. In the series of experiments, the input signal - photon number was adjusted using calibrated neutral density filters to the values 1,3 , $10, \ldots$ up to 10000 photons per event. The avalanche current response pulses were recorded, stored and off-line processed using the custom designed software package. The times when the avalanche current exceeded the pre-selected level were evaluated. The results are summarized in figure 1. The risetime - the time interval between signal crossing of the $10 \mathrm{mV}$ and $1.5 \mathrm{~V}$ levels is plotted along with the detection delay related to the trigger level of $10 \mathrm{mV}$. The outliners in the risetime for the average photon numbers of 3 and 10 photons per event are caused by the photon counting statistics - Poisson distribution. The averages of 50 records are plotted for each photon number. The individual values spread is about $35 \mathrm{ps}$ for photon numbers in the range of 1 to 100 photons and drops down to about 15 ps for higher photon numbers. The dependence of the detection delay and pulse risetime was studied in an broad dynamical range from single photons up to 10 thousands photons in one optical pulse hitting the detector active area. The total risetime difference is $0.6 \mathrm{~ns}$ for the photon number range of 1 to 10000 .

The detection delay for various photon numbers has been tested for two different optical configurations. In the first configuration the detected photons were absorbed within a small area close to the sensitive area center. In the second configuration the detected photons were absorbed uniformly over the active area. The results has been comparable in terms of experimental errors described above.

In tested detection structure the avalanche current risetime contains information about photon number detected in the dynamical range 1 photon to more than 3000 photons per pulse. This is valid for optical pulses which are much shorter in comparison to the avalanche current risetime. The avalanche pulse risetime measurement permits to determine the photon number in involved in the detection process and to determine the detection time walk.

This research has been supported by numerous grants provided by the Grant Agency of the Czech Republic, Ministry of Education of the Czech Republic and the international grant agencies, as well. The recent publication has been supported by ME08063.

\section{References}

[1] S. Cova, et al, Journal of Modern Optics 51 (9-10), pp. 1267 - 1288, 2004.

[2] R. H. Haitz, J. of Appl. Phys. 35, p. 1370, 1964.

[3] G. Kirchner, et al, "Time Walk Compensated SPAD: Multiple Photon Versus Single Photon Operation”, in Proc. SPIE 3218, pp. 106-112, 1997.

[4] I. Prochazka, et al., Journal of Modern Optics 51 (9-10), pp. 1289 - 1313, 2004.

[5] I. Prochazka, et al., Journal of Modern Optics 54 (2-3), pp. 141 - 150, 2007. 